\title{
Incidence of Urinary Tract Infections and Allergic Conditions among Type 2 Diabetes Mellitus Patients in Rural Hospital Clinics in Sri Lanka
}

\author{
JGA Sachinthana ${ }^{1}$, GM Kariyawasam ${ }^{1}$, EMDTEdirisinghe ${ }^{1}$, HSN Peiris ${ }^{1}$, DARK Dayarathna ${ }^{2}$, \\ FM Mowlana ${ }^{1}$ and JMKB Jayasekara ${ }^{1 *}$
}

${ }^{1}$ Department of Medical Laboratory Science, Faculty of Allied Health Sciences, Sir John Kotelawala Defense University, Sri Lanka ${ }^{2}$ District General Hospital, Ampara, Sri Lanka

*Corresponding author: JMKB Jayasekara, Department of Medical Laboratory Science, Faculty of Allied Health Sciences, Sir John Kotelawala Defense University, Sri Lanka

\begin{abstract}
Background: Urinary tract infections (UTI) and allergic conditions in individuals with diabetes mellitus (DM) may evolve to serious complications unless managed properly. Early diagnosis and evaluation of these conditions of DM patients are important to maintain the quality of life of the patients. The objective of the current study was to identify the incidence of UTIs among type 2 DM patients.

Method: A retrospective cross-sectional study was conducted on 260 type 2 DM patients, who participated in a diabetic clinic conducted in the District General Hospital, Ampara. The study was conducted for a period of one month. Information like demography, history of UTI reported in the last 12 months, glycemic control of patients and other relevant details were collected via a questionnaire. In addition, Urine Full Report (UFR) of spot urine sample and Full Blood Count (FBC) investigations were performed in the hospital laboratory and analyzed.

Results: The incidence of UTI in the study population was $27.2 \%(n=72)$. UFR showed $25 \%$ of the study population had evidence of UTI (presence of 10 or more puss cells/ high power field of the microscope). A significant association was noticed between the incidence of UTI and the gender $(p<0.01)$. High incidence of UTI was observed in females $(81.9 \%)$ with a relative risk of $2.0(95 \% \mathrm{Cl} 0.208,0.701)$. Moreover, the study showed a statistically significant association between the incidence of UTI ( $p<0.05$, relative risk, $2.5(95 \% \mathrm{Cl}=1.053,5.936))$ and female patients having poor glycemic control. A statistically significant association was also shown between the incidence of UTI and the duration of DM $(p<0.05)$ which was seen to be greater than 6.5 years. The incidence of allergic conditions in the study population was $25.8 \%(n=67)$.
\end{abstract}

Conclusion: Female subjects are more affected with UTI than male subjects in the study population. In addition, a significant association was found between the prevalence of UTI and female patients with poor glycemic control. Duration of DM also showed significant association with UTI.

\section{Keywords}

Diabetes mellitus, Urinary tract infections, Allergic conditions, Urine full report

\begin{abstract}
Abbreviations
ASB: Asymptomatic Bacteriuria; DM: Diabetes Mellitus; FBS: Fasting Blood Sugar; FBC: Full Blood Count; SPSS: Statistical Package for the Social Sciences; UFR: Urine Full Report; UTI: Urinary Tract Infections
\end{abstract}

\section{Introduction}

Diabetes mellitus (DM) is a group of metabolic disorders commonly presenting with episodes of hyperglycemia and glucose intolerance. This can be caused due to a lack of insulin, a defective insulin action or both. Classification of DM is based on its aetiology and clinical presentation. Many people are afflicted with type 2 diabetes where around $90 \%$ of DM patients in the world are living with this type. The major contributing factor to type 2 diabetes is excess body weight and lack of physical activity [1]. One in five adults in Sri Lanka has either diabetes or pre-diabetes and one-third of the patients affected by diabetes are undiagnosed [2].

Citation: Sachinthana JGA, Kariyawasam GM, Edirisinghe EMDT, Peiris HSN, Dayarathna DARK, et al. (2018) Incidence of Urinary Tract Infections and Allergic Conditions among Type 2 Diabetes Mellitus Patients in Rural Hospital Clinics in Sri Lanka. Int J Diabetes Clin Res 5:097. doi.org/10.23937/23773634/1410097

Accepted: November 05, 2018: Published: November 07, 2018

Copyright: (C) 2018 Sachinthana JGA, et al. This is an open-access article distributed under the terms of the Creative Commons Attribution License, which permits unrestricted use, distribution, and reproduction in any medium, provided the original author and source are credited. 
Out of the people afflicted with diabetes, greater frequency of infections is caused by the hyperglycemic environment that favors immune dysfunction (e.g. damage to neutrophil function, depression of the antioxidant system, and humoral immunity), micro-and macro-angiopathies, neuropathy, and a decrease in the antibacterial activity of urine. The main risk factors for UTI in DM are inadequate glycemic control, duration of DM, diabetic microangiopathy, impaired leukocyte function, recurrent vaginitis, and anatomical and functional abnormalities of the urinary tract. The spectrum of UTI in these patients ranges from asymptomatic bacteriuria to lower UTI (cystitis), pyelonephritis, and severe urosepsis. Serious complications of UTI, such as emphysematous cystitis and pyelonephritis, renal abscesses and renal papillary necrosis, are encountered more frequently in diabetes than in the general population. Long-term DM patients who are undergoing recurrent UTI may encounter renal failure [3].

Underlying immune mechanisms of allergic conditions and DM suggest that allergy should be less common in DM patients in the general population [4]. An imbalance towards $T$ helper 1 lymphocyte expression would favour the development of auto-immune diseases such as some types of diabetes thought to be mediated by $T$ helper 1 cells and inhibit atopy and asthma mediated by T helper 2 cells [4]. Thus, early diagnosis and evaluation of UTI and allergic conditions of the DM patients are important. Therefore, this study will be helpful to upgrade the quality of life of DM patients in Sri Lanka.

\section{Methods}

A retrospective and descriptive cross-sectional study was conducted among the patients who were registered in a diabetes clinic in General Hospital, Ampara, Sri Lanka. Two hundred and sixty patients (260) were selected, aged between 18 to 70 years. Patients with gestational DM were excluded because their number was very less $(n=7)$ within the study population and it was not sufficient for further analysis. Diagnosed UTI patients and patients with prevailing allergic conditions before the onset of DM were also excluded. Patients who refused informed consent were not considered for this study. Patients were interviewed and incidence of clinically presented UTI and allergic conditions in the last 12 months period were collected by investigating their clinical records. Allergic conditions such as asthma, insulin allergy, other drug allergies, urticaria/rash, hay fever and eczema were mainly considered. Demo- graphic details, baseline symptoms and clinical history including glycemic control of all the patients were collected through their clinic records and interviewer-administered questionnaire.

Fifteen (15) $\mathrm{ml}$ of spot urine was collected from patients and UFR was performed in order to confirm the findings. All the UFR investigations were performed at the biochemistry laboratory of District General Hospital, Ampara. The parameters including pus cells, organisms, red cells, epithelial cells, crystals and casts were analyzed. In addition, $4 \mathrm{ml}$ of blood was collected from 50 patients' cohort and $\mathrm{FBC}$ was performed using the Mindray BC5800 fully automated hematological analyzer. All the parameters including WBC count and eosinophil count were analyzed at the hematology laboratory of District General Hospital, Ampara. Leishman stained blood smears were observed microscopically to confirm the automated count and to identify the absolute eosinophil count of the patients. The spot urine analysis and the $\mathrm{FBC}$ analysis were performed exclusively for this study.

Monthly fasting blood sugar (FBS) value of the patients was collected over the last 12 months period and the mean was calculated. They were categorized into two groups as mean FBS less than $126 \mathrm{mg} / \mathrm{dl}$ (well controlled) and mean FBS more than or equal to $126 \mathrm{mg} / \mathrm{dl}$ (poorly controlled). In the second part of the study, the total number of patients who had clinically presented with allergic conditions during the last 12 months period was analyzed.

Associations among the variable groups were analyzed by using the Statistical Package for the Social Sciences (SPSS) version 22. The ethical approval for the study was obtained from Ethical review committee, Faculty of Medicine, Sir John Kotelawla Defence University, Sri Lanka.

\section{Results}

Out of the two hundred and sixty type 2 patients having diabetes who participated in the study, 30.4\% were male patients and $69.6 \%$ female. The mean age of the study population was 59 years. The mean age of the female patients was $57(n=181)$ years while 62 years $(n$ $=79$ ) for males. Table 1 summarizes the demographics, duration of DM and the incidences of UTI and allergic conditions in the study population. Approximately $30 \%$ of the patients were employed and $70 \%$ were unemployed. Majority of the study population was from the Sinhalese community (97\%).

Table 1: Demographics.

\begin{tabular}{|l|l|l|l|}
\hline & Male $(\mathbf{n}=\mathbf{7 9})$ & Female $(\mathbf{n}=\mathbf{1 8 1})$ & Total $(\mathbf{n}=\mathbf{2 6 0})$ \\
\hline Mean Age (years) & 62.35 & 57.22 & 58.78 \\
\hline Duration of DM (years) & 7.25 & 5.75 & 6.50 \\
\hline Incidence of UTI & 13 & 59 & 72 \\
\hline Incidence of allergic conditions & 20 & 47 & 67 \\
\hline
\end{tabular}


Table 2: Descriptive statistics of mean FBS categories and total patients $(n=260)$.

\begin{tabular}{|l|l|l|l|}
\hline & $\begin{array}{l}\text { Mean FBS } \mathbf{2} \mathbf{1 2 6} \mathbf{~ m g / d l} \\
(\mathbf{n}=\mathbf{1 5 2}, \text { poorly controlled) }\end{array}$ & $\begin{array}{l}\text { Mean FBS }<\mathbf{1 2 6} \mathbf{~ m g / d l} \\
\mathbf{( n = 1 0 8 , ~ w e l l ~ c o n t r o l l e d ) ~}\end{array}$ & $\begin{array}{l}\text { Total patients } \\
(\mathbf{n}=\mathbf{2 6 0})\end{array}$ \\
\hline Mean & 108.2 & 62.35 & 141.198 \\
\hline Median & 109.6 & 64.00 & 135.35 \\
\hline Mode & 108.3 & 59 & 108.3 \\
\hline Std. Deviation & 11.0 & 11.151 & 41.3577 \\
\hline Minimum & 79.4 & 18 & 79.4 \\
\hline Maximum & 125.6 & 87 & 394 \\
\hline
\end{tabular}

Table 3: Incidence of UTI according to the gender and glycemic control groups $(n=260)$.

\begin{tabular}{|l|l|l|l|l|}
\hline & & $\begin{array}{l}\text { Mean FBS < 126 mg/dl } \\
\text { (well controlled) }\end{array}$ & $\begin{array}{l}\text { Mean FBS } \mathbf{1 2 6} \text { mg/dl }(\mathbf{n}=152, \\
\text { poor controlled }\end{array}$ & Total $(\mathbf{n}=\mathbf{2 6 0})$ \\
\hline UTI Present & Male & $8(3.07 \%)$ & $5(1.93 \%)$ & $13(5.0 \%)$ \\
\hline & Female & $24(9.24 \%)$ & $35(13.46 \%)$ & $59(22.7 \%)$ \\
\hline UTI Absent & Male & $31(11.94 \%)$ & $35(13.46 \%)$ & $66(25.4 \%)$ \\
\hline & Female & $45(17.29 \%)$ & $77(29.61 \%)$ & $122(46.9 \%)$ \\
\hline TOTAL & & $108(41.54 \%)$ & $152(58.46 \%)$ & $260(100 \%)$ \\
\hline
\end{tabular}

Table 4: Association between prevalence of UTI and the duration of DM.

\begin{tabular}{|l|l|l|l|}
\hline \multicolumn{1}{|l|}{$\begin{array}{l}\text { UTI Absent } \\
\text { less than 6.5 years }\end{array}$} & $127(48.8 \%)$ & $37(14.2 \%)$ & Total \\
\hline $\begin{array}{l}\text { Duration of DM } \\
\text { more than or equal } \\
\text { 6.5 years }\end{array}$ & $61(23.5 \%)$ & $35(13.5 \%)$ & $96(36.9 \%)$ \\
\hline Total & $188(72.3 \%)$ & $72(27.7 \%)$ & $260(100.0 \%)$ \\
\hline
\end{tabular}

The descriptive statistics of the monthly fasting blood sugar (FBS) value of the patients collected over the last 12 months period of each group is depicted in Table 2.

Incidence of clinically presented UTI in the study population was given in Table 3 for male and female groups respectively.

As per the results of the present study, a statistically significant association between the incidence of UTI and the gender $(p=0.007)$ was observed. Among 72 patients who had UTI during the period of last 12 months, 59 (81.9\%) were female. The relative risk of getting UTI for female patients than male patients was 1.981 (95\% $\mathrm{Cl} 1.155,3.397)$. The odds ratio female/male was 2.45 $(95 \% \mathrm{Cl}=1.255,4.803)$. A statistically significant association between the incidence of UTI and the gender ( $p=0.021$ ) was observed from patients with mean FBS more than or equal $126 \mathrm{mg} / \mathrm{dl}$. Among 40 patients who had UTI during the period of last 12 months, 35 (87.5\%) were females. The relative risk of getting UTI for female patients with mean FBS more than or equal $126 \mathrm{mg} / \mathrm{dl}$ was $2.5(95 \% \mathrm{Cl}=1.053,5.936)$. The odds ratio female/ male was $0.314(95 \% \mathrm{Cl}=0.113,0.870)$. For the patients with mean FBS less than $126 \mathrm{mg} / \mathrm{dl}$, the association between the prevalence of UTI and the gender was not statistically significant.

Out of all the UTI patients, $56.9 \%$ had a UTI once and 43.1\% had UTI more than once during the 12 months period. In addition, UFR was analyzed to identify the point prevalence of UTI among the study population.
$25 \%$ of patients had pus cells count greater than 10 per high power field and two organisms or above.

We categorized the population into two according to the known DM duration. With reference to the results of the present study, a statistically significant association between the prevalence of UTI and the duration of DM ( $p=0.016$ ) was observed (Table 4). Among the 188 patients who had no UTI during the last 12 months, 127 (67.6\%) were having a duration of DM less than 6.5 years. The odds ratio of less than 6.5 years/more than or equal 6.5 years was $1.969(95 \% \mathrm{Cl}=1.132,3.472)$.

In the study population, the total number of patients who had allergic conditions during the period of the last 12 months was 67 (25.8\%). Among them, 47 were female patients and 20 were male patients (Table 3 ). The prevalence of allergic conditions was cross-tabulated according to gender. The association between the prevalence of allergic conditions and gender was not statistically significant ( $p=0.912$ ).

In the performed $\mathrm{FBC}$, all the parameters including WBC count and eosinophil count were analyzed. Among 50 FBCs, $8 \%$ of the patients had high eosinophil count and $12 \%$ of the patients had high WBC count. The association between eosinophil count and the mean FBS categories were not statistically significant $(p=0.801)$. Similarly, the association between WBC count and the mean FBS categories were not statistically significant ( $p$ $=0.233$ ). 


\section{Discussion}

In the current study, type 2 DM patients were considered and high incidence of clinically presented UTI $(27.7 \%)$ was observed in the study population which was female dominant. This was further confirmed by the UFR results performed using the spot urine samples of the patients. Around $25 \%$ of the study population was having evidence of UTI during the period of urine sample collection.

Similar studies have been reported with a high incidence of UTI among DM patients. In 2009, a study concluded that the prevalence of lower urinary tract infections in South Indian type 2 diabetic subjects was $42.8 \%(n=495)$ [5]. Prevalence of UTI and antimicrobial susceptibility among people living with diabetes with controlled and uncontrolled glycemia in Kuwait in 2015, showed $35 \%$ ( $n=252$ ) of the samples were positive for uropathogens [6].

This is not a phenomenon observed only in the Asian countries. In the United States, the prevalence of UTI was $23.5 \%$ ( $n=235)$ for 1000 type 2 DM patients [7]. Also, the study reported an increased prevalence of asymptomatic bacteriuria (ASB) in DM patients, with estimates ranging from $8 \%-26 \% .8$ in the present study we considered only clinically reported cases, thus the actual percentage of UTI may be high than the reported cases.

Similar to the previous studies conducted, high incidence of UTI was observed in female patients in the Sri Lankan population. Among the 72 patients who had UTI during the 12 months period, over $80 \%$ were females $(p<0.01)$. Relative risk was observed as $1.9(95 \%$ $\mathrm{Cl} 0.208,0.701)$. Janifer, et al. showed women (47.9\%) had a significantly higher prevalence of urinary tract infection than men (34.1\%) [5]. In another study, females showed a much higher prevalence of UTI than males in both controlled and uncontrolled glycemic groups (88.5\% and $11.5 \%$, respectively, $P<0.0001$ ) [6]. The hospital-based study showed three folds high risk for females in getting UTI than in male DM patients [8]. Grandy, et al. also identified women were 4.2 times more likely than men to have at least one UTI, after taking the diabetes status and age into consideration in his study $(p<0.001)$ [7]. A very significant finding was observed in the study conducted by Nitzan, et al. who reviewed that ASB was more prevalent in women, due to the short urethra that is in proximity to the warm, moist, vulvar, and perianal areas that are colonized with enteric bacteria. ASB increases with age and is also associated with urinary tract abnormalities or foreign bodies (urethral catheters, stents, etc). The point prevalence of ASB was higher in both women and men. It was also higher in patients with a longer duration of diabetes and was not associated with glycemic status, as evaluated by $\mathrm{HbA} 1 \mathrm{c}$ [9].

In the current study, we estimated the glycemic control by analyzing mean FBS levels for the past 12 months. Patients' HbA1c levels were not available as the hospital does not conduct such investigations. The population was also under middle to low-income group; therefore, they are unable to do expensive investigations from private laboratories. Statistically significant association was observed between prevalence of UTI and the gender $(p<0.05)$ in the group of uncontrolled DM which was not shown in the controlled DM group. The relative risk of getting UTI for female than in male patients in this category was $2.5(95 \% \mathrm{Cl} 1.053,5.936)$.

According to a South Indian study, poor glycemic control was significantly associated with UTI in both sexes $(P=0.0005)$ [5]. A study in Kuwait showed that most UTI cases occurred in the uncontrolled glycemic group (197 patients) than the controlled glycemic group (55 patients). But in contrast with the South Indian study, females in Sri Lanka showed a much higher prevalence of UTI than males in both controlled and uncontrolled glycemic groups $(88.5 \%$ and $11.5 \%$, respectively, $\mathrm{P}<$ 0.0001 ) [6]. In a recent paper, Nitzan, et al. reviewed the multiple potential mechanisms unique to diabetes that may contribute to the increased risk of UTI in people living with diabetes. Higher glucose concentrations in urine may promote the growth of pathogenic bacteria. However, several studies did not find an association between HbA1c levels, which serves as a proxy for glycosuria [9].

Low incidence of UTI was noticed in patients with duration of DM less than 6.5 years in the current study. Among the 188 patients who had no UTI during the past 12 months, 127 (67.6\%) were having a duration of DM less than 6.5 years $(p=0.016)$. Janifer, et al. also stated that the rates of the incidence of UTI increased with the increasing duration of diabetes. Further, he said, the prevalence of UTI was not very marked until the age of 45 years and thereafter, a significant increase was seen in both men and women [5]. A study in Kuwait reported that, in DM patients, specific risk factors for UTI are usually the duration of diabetes and the presence of long-term complications, such as neuropathy, rather than current glucose control [6]. This might be due to the fact that bladder dysfunction occurs in $26 \%-85 \%$ of people living with diabetes, depending on age, extent of neuropathy and duration of diabetic disease, and thus this should be considered in all people troubled with diabetes with UTI [9]. In contrast, a cross-sectional study conducted in Sudan showed no association between duration of DM and UTI, using the logistic regression. Therefore, the proper screening of DM patients for UTI should be conducted especially in the poorly controlled and long-standing DM patients. This will help upgrade the quality of life of DM patients.

The second section of the study focused on the allergic conditions among type 2 Diabetes mellitus patients in the study population. The study reported it was 
$25.8 \%$ and comparatively high. However, most of the studies done by the other countries on type 2 diabetes had limited evidence to support and describe the different effects of allergic conditions on type 2 DM patients. Underlying immune mechanisms of allergic conditions and DM had suggested that allergy should be less common in DM patients in the general population [4]. In 2009, a study was conducted on the increase in the prevalence of allergy-like respiratory symptoms in insulin-dependent DM patients, however, there was a contrasting decrease in positive skin prick tests. Although in the diabetic group, $56 \%$ of the patients reported allergy-like respiratory symptoms, only $22 \%$ were found to have positive skin tests. In the control group, $22 \%$ had allergic complaints and skin prick tests were positive in $34 \%$ of the cases [10]. Another study conducted in Turkey, on the frequency of atopy and allergic disorders among adults with type 2 diabetes mellitus. According to the results of the skin-prick test, the rate of positive response to at least one allergen was not significantly different in diabetes group (29.2\%) and in the control group (31.3\%). The rate of physician-diagnosed asthma was similar in both groups. There was no difference between the two groups based on the answers to questions about asthma and other allergic diseases such as allergic rhinitis, eczema, and drug allergy [11].

\section{Conclusions}

The study concluded the incidence of UTI in the study population was high and it was about $27.7 \%$ as shown by the spot analysis. The study also concludes that the female subjects are more vulnerable to getting UTI than their male counterparts. In addition, a significant association was found between the incidence of UTI and the gender in patients having poorly controlled DM. Furthermore, patients having DM for longer durations were seen to have higher incidences of UTI in the study population.

\section{Acknowledgements}

We wish to acknowledge the Director, Deputy Director, Consultant physicians, Medical officers, chief nursing officers and nursing staff of the Diabetic clinic and Diabetic Centre, and the laboratory technologists and laboratory assistants of the Hematology and Clinical
Biochemistry laboratories of District General Hospital, Ampara.

\section{References}

1. WHO (2015) Diabetes.

2. Katulanda P, Constantine GR, Mahesh JG, Sheriff R, Seneviratne RD, et al. (2008) Prevalence and projections of diabetes and pre-diabetes in adults in Sri Lanka-Sri Lanka Diabetes, Cardiovascular Study (SLDCS). Diabet Med 25: 1062-1069.

3. Brusch, John L (2015) Urinary Tract Infections in Diabetes Mellitus.

4. Dales R, Chen Y, Mei Lin, Karsh J (2005) The Association between Allergy and Diabetes in the Canadian Population: Implications for the Th1-Th2 Hypothesis. Eur J Epidemiol 20: 713-717.

5. Janifer J, Geethalakshmi S, Satyavani K, Viswanathan V (2009) Prevalence of lower urinary tract infection in South Indian type 2 diabetic subjects. Indian J Nephrol 19: 107111.

6. Sewify M, Nair S, Warsame S, Murad M, Alhubail A, et al. (2016) Prevalence of Urinary Tract Infection and Antimicrobial Susceptibility among People living with diabetes with Controlled and Uncontrolled Glycemia in Kuwait. J Diabetes Res.

7. Grandy S, Fox KM, Hardy E (2013) Prevalence and recurrence of urinary tract and genital infections among adults with and without type 2 diabetes mellitus in the general population: a longitudinal cohort study. J Diabetes Res Clin Metab 2: 5 .

8. Czaja CA, Rutledge BN, Cleary PA, Chan K, Stapleton AE, et al. (2009) Urinary Tract Infections in Women with Type 1 Diabetes Mellitus: Survey of Female Participants in the Epidemiology of Diabetes Interventions and Complications Study Cohort. J Urol 181: 1129-1134.

9. Nitzan O, Elias M, Chazan B, Saliba W (2015) Urinary tract infections in patients with type 2 diabetes mellitus: review of prevalence, diagnosis, and management. Diabetes Metab Syndr Obes 8: 129-136.

10. Erdenen F, Altungolu E, Boz M, Celik S, Ozbal N, et al. (2009) Increase in prevalence of allergy like respiratory symptoms in IDDM patients contrasting with a decrease in positive skin prick tests. Bull Soc Sci Med Grand Duche Luxemb 135-140.

11. Duran C, Ediger D, Ersoy C, Coskun NF, Selimoglu H, et al. (2008) Frequency of atopy and allergic disorders among adults with Type 1 diabetes mellitus in the southern Marmara region of Turkey. J Endocrinol Invest 31: 211-215. 